\title{
Developmental Trajectories of Perceived Friendship Intimacy, Constructive Problem Solving, and Depression from Early to Late Adolescence
}

\author{
M. H. W. Selfhout • S. J. T. Branje • W. H. J. Meeus
}

Published online: 21 October 2008

(C) The Author(s) 2008. This article is published with open access at Springerlink.com

\begin{abstract}
This study examined friendship types in developmental trajectories of perceived closeness and balanced relatedness. In addition, differences between friendship types in the development of constructive problem solving and depression were examined. Questionnaire data of five annual waves were used from two adolescent cohorts (cohort 1: $M=12.41$ years; cohort 2: $M=16.37$ years). Growth Mixture Modeling revealed two developmental trajectories in cognitive representations of perceived friendship intimacy: interdependent and disengaged friendships. Adolescents in interdependent friendships were characterized by high perceived closeness and balanced relatedness across adolescence. Furthermore, adolescents in disengaged friendships had lower levels of and smaller increases in constructive problem solving. Girls in disengaged friendships showed smaller increases in balanced relatedness and higher levels of depression than boys in disengaged friendships and adolescents in interdependent friendships.
\end{abstract}

Keywords Adolescence · Development · Friendship intimacy types · Growth mixture modeling · Depression . constructive problem solving

Intimate friendships first seem to appear during early adolescence, primarily because the ability to balance

M. H. W. Selfhout $(\bowtie) \cdot$ S. J. T. Branje $\cdot$ W. H. J. Meeus Research Centre Adolescent Development, Utrecht University, P.O. Box 80140, 3508 TC, Utrecht,

The Netherlands

e-mail: M.Selfhout@uu.nl

S. J. T. Branje

e-mail: S.Branje@uu.nl

W. H. J. Meeus

e-mail: W.Meeus@uu.nl closeness and individuality in friendships does not emerge until this period (Selman 1990). Intimacy may be critical in differentiating friendship types in adolescence. Adolescents who perceive less intimate friendships may develop less constructive ways to resolve conflicts in friendships (e.g., Shulman et al. 1997a) and may be at risk for developing depression (e.g., Shulman and Laursen 2002). However, more research is needed to examine different developmental trajectories of perceptions of friendship intimacy, as well as differences between friendship types in developmental trajectories of conflict resolution and depression. The current study examined whether different friendship types could be distinguished based on developmental trajectories of perceived friendship intimacy from early to late adolescence (for boys and girls separately). Furthermore, differences between these friendship intimacy types in the developmental trajectories of perceived constructive problem solving and depression across adolescence were examined.

\section{Perceptions of Friendship Intimacy in Adolescence}

Several developmental theories suggest that a balance between two characteristics of intimacy in relationships is crucial for optimal development: closeness and individuation. Closeness refers to the interpersonal processes whereby friends feel bonded to each other (Reis and Shaver 1988). Individuation refers to the process by which social individuals become differentiated from the other and has been described as a hallmark of the developing adolescent (Sullivan 1953). At least three theoretical perspectives emphasize a role of both closeness and individuality. Attachment theory (Bowlby 1973) suggests that optimal adaptation within close relationships involves use of a 
partner not only as a source of closeness, but also as a secure base for individual exploration. In a similar vein, theories concerning connectedness and individuation (Grotevant and Cooper 1986) suggest that normative development across adolescence is characterized by maintaining close relationships with parents and peers while at the same time increasing individuation. Individuation emerges gradually during adolescence, as adolescents strive to distinguish themselves from both parents and peers (Grotevant and Cooper 1998). Furthermore, theories dealing with parenting practices (Baumrind and Black 1967) suggest that providing warmth as well as encouraging children's psychological autonomy is associated with positive developmental outcomes.

Although these theories primarily deal with parentadolescent and partner relationships, closeness and individuation also seem to characterize cognitive representations or perceptions of intimacy in adolescent friendships (Selman 1990). Selman defined two developmental levels of intimacy from late childhood to late adolescence. First, during late childhood and early adolescence, closeness is expressed by sharing experiences with regard for the other's opinion but individuality is negotiated in a persuasive manner, with a focus on individual needs. Therefore, perceived closeness and individuality are still relatively low in early adolescence. From middle adolescence onwards, individuality is negotiated through collaborative strategies oriented towards integration of needs of the self and the other, while at the same time feelings of closeness towards best friends increase.

Shulman and Knafo (1997) partially adopted Selman's developmental model in that they also proposed a transition in friendship intimacy from a focus on the self to a focus on both the self and the friend middle adolescence. However, they identified at least two friendship types that differ in this development: interdependent and disengaged friendships. They focused on one important aspect of perceived individuality in adolescent friendships: balanced relatedness, or the extent to which an adolescent feels that his or her friend accepts his or her opinions, wishes, and needs (Shulman and Knafo 1997).

Already in early adolescence, adolescents in disengaged friendships are thought to experience less closeness towards their friends than adolescents in interdependent friendships, because they view dependence on friends as a sign of weakness. During middle adolescence, interdependent friends develop an awareness of each other's needs, and therefore feel that their individuality is accepted more by their friends than adolescents in disengaged friendships. In addition, interdependent friends remain more emotionally close to each other than disengaged friends. In late adolescence, both perceived closeness and balanced relatedness remain higher for adolescents in interdependent friendships compared to adolescents in disengaged friendship. Thus, according to this model, adolescents in interdependent friendships perceive higher closeness from early adolescence onwards and higher balanced relatedness from middle adolescence onwards compared to adolescents in disengaged friendships.

A series of experiments (Shulman 1993; Shulman and Knafo 1997; Shulman and Laursen 2002) involving a joint card sorting task for adolescent friends classified early and middle adolescents who improved much when interacting and also collaborated more than other friendship dyads as interdependent friends. Those low on these characteristics were classified as disengaged friends. Around half were interdependent friends and the other half were disengaged friends. Another study used cluster analyses on perceived friendship characteristics and found adolescents in disengaged friendships based on reports of low closeness, intimacy, companionship, and satisfaction, whereas three other groups were found with higher scores on these dimensions (Way et al. 2001). One indicator of perceived closeness, namely perceived commitment, most clearly differentiates between disengaged and other types of friendship and differences in these friendship types in adolescent development, presumably because it is a relatively strong indicator of closeness compared to other indicators (Way et al. 2001). Therefore, we will focus on perceived commitment when examining friendship types.

Thus, taken together, cross-sectional evidence suggests that at least two friendship types exist in adolescence: interdependent friendships, with higher levels of commitment and balanced relatedness, and disengaged friendships, with lower levels of commitment and balanced relatedness. Although the developmental trajectories of these friendship types have been suggested to differ, the actual development of friendship intimacy across adolescence has not been examined yet. Thus, to provide more insight in the way friendship types develop across adolescence, research is needed that examines friendship intimacy from early to late adolescence.

Contradictory evidence exists about gender differences in the proportions of adolescents in interdependent and disengaged friendships. Some studies showed similar proportions of early adolescent boys and girls in the two friendship types (Shulman 1993, 1995; Shulman and Knafo 1997), others found that boys were more likely to be in the disengaged group than girls (Shulman and Laursen 2002; Way et al. 2001). Furthermore, girls have been shown to perceive higher commitment (e.g., Cheng and Chan 2004; Furman and Buhrmester 1992) and higher balanced relatedness (Shulman and Knafo 1997) in their best friendships than boys, suggesting that girls more frequently have interdependent friendships than boys do, and boys more frequently have disengaged friends than girls do. 


\section{Friendship Types and Constructive Problem Solving}

Over the course of adolescence, adolescents increasingly learn to use constructive problem solving, or to negotiate between their own views and views of others, during conflicts with best friends (Laursen 1993). A meta-analysis of problem solving styles showed that constructive problem solving is one of the most commonly used tactics to resolve conflicts in friendships, and that constructive problem solving increases from childhood to adulthood (Laursen et al. 2001). Developmental models of friendship intimacy propose that differences in development of perceived commitment and balanced relatedness are mainly expressed in different developmental trajectories of constructive problem solving during conflicts (Selman 1990; Shulman and Laursen 2002). From middle adolescence onwards, adolescents in interdependent friendships are thought to actively negotiate between their own ideas and opinions and those of their best friends and to increase in constructive problem solving from middle adolescence onwards, when friends need to start recognizing each others' individual opinions, ideas, and needs. In contrast, adolescents in disengaged friendships are thought to be less likely to use constructive ways of problem solving and choose to blame each other for mistakes without taking responsibility, or to avoid talking about the conflict all together (Shulman and Laursen 2002). Consistent with this view, seventeen year-old adolescents in interdependent friendships have been shown to use more constructive problem solving to resolve conflicts than adolescents in disengaged friendships (Shulman and Laursen 2002). Thus, adolescents in interdependent and disengaged friendships differ in mean levels of constructive problem solving in middle adolescence. However, it remains unclear whether these differences between friendship types can be observed in the developmental trajectory of constructive problem solving from early to late adolescence.

\section{Friendship Types and Adolescent Depression}

Whereas for girls depression typically increases from early adolescence into adulthood, for boys depression decreases across adolescence (Rudolph et al. 2000). The previously discussed attachment theory (Bowlby 1973), theories regarding the connectedness-individuation link (Grotevant and Cooper 1986), and theories dealing with parenting practices (Baumrind and Black 1967) emphasize that positive developmental outcomes occur when commitment and individuation are balanced in relationships. In a similar vein, it has also been suggested that adolescents in disengaged friendships experience more emotional problems than adolescents in interdependent friendships (Shulman and Knafo 1997).

Several processes might explain why depression and low quality friendships, such as disengaged relationships, may be linked. First, adolescents with more depressive feelings typically have lower levels of social skills (Engels et al. 2002), making it more difficult for them to maintain a friendship in which both commitment and balanced relatedness are high. Furthermore, adolescents with depression may elicit more negative responses from friends, who therefore evaluate them as less likeable and less attractive, and engage in fewer positive behaviors with them (Coyne and Downey 1991; Hale 2001; Rudolph et al. 2000). This may result in lower perceived commitment and balanced relatedness by the depressed adolescent. In addition, lower quality friendships might lead to more loneliness and depression, since adolescents in such friendships may experience low social support when facing emotional problems (Prinstein et al. 2005b).

Adolescents in disengaged friendships concurrently reported higher levels of depressive symptoms than adolescents in interdependent friendships (Way et al. 2001). Furthermore, high commitment and high balanced relatedness can be viewed as two indicators for high friendship quality, and cross-sectional (Kiesner et al. 2003) and over-time (Prinstein et al. 2005a; Stice et al. 2004) associations between low perceived friendship quality and depression in adolescence have been shown. Nevertheless, differences between friendship types in developmental trajectories of depression remain unclear.

Further, girls' emotional states may be more affected by the quality of their friendship than boys' emotional states are (Prinstein et al. 2005a; Rudolph 2002; Rudolph and Hammen 1999). For example, adolescent girls were found to report more depressive symptoms after experiencing interpersonal stress than boys (Rudolph 2002) and are suggested to be more sensitive to changes in friendship quality compared to boys (Rose et al. 2007). Therefore, differences in depression between friendship types may be more pronounced for girls than for boys. Finally, adolescents with stable friendships have been found to have less depression than adolescents with low stable friendships (Prinstein et al. 2005a). We will therefore control for differences in depression between stable versus non-stable friendships when examining differences between friendship types in depression.

This study will examine the following research questions:

1. Can adolescent friendships be classified as interdependent and disengaged friendships based on developmental trajectories of perceived commitment and balanced relatedness? Based on the theoretical model of Shulman and Knafo (1997), the first class is expected to show 
higher perceived commitment from early adolescence onwards and higher perceived balanced relatedness from middle adolescence onwards compared to the second class. We will explore gender differences in these developmental trajectories and in the distributions of these classes.

2. To what extent do adolescents in interdependent and disengaged friendships differ in their development of constructive problem solving during conflicts in their friendship? We will explore whether adolescents in interdependent friendships show higher mean levels and a stronger increase in constructive problem solving from middle adolescence onwards compared to adolescents in disengaged friendships.

3. To what extent do adolescents in interdependent and disengaged friendships differ in their development of depression? We will explore whether adolescents in disengaged friendships show higher mean levels and a stronger increase in depression from early to late adolescence compared to adolescents in interdependent friendships.

\section{Method}

\section{Participants}

Participants in this study were 911 adolescents of the young and old adolescent cohort participating in the CONflict And Management Of RElationships study (CONAMORE) (Meeus, W., Akse, J., Branje, S., ter Bogt, T., Engels, R., Finkenauer, C., et al. (2002). Codebook CONAMORE: Conflicts and management of relationships. Unpublished manuscript). CONAMORE is an ongoing longitudinal study that examines the relationships of Dutch adolescents with parents and peers as well as their emotional states. In the current study, data were used from target adolescents who filled out questionnaires on all five waves with a oneyear interval. Of the 911 adolescents, 48.6\% were boys and $51.4 \%$ were girls. The mean age of the first cohort at the first wave was $12.41(\mathrm{SD}=0.58)$ and of the second cohort at the first wave was $16.37(\mathrm{SD}=0.84)$. The first age cohort was followed from age 12 to age 16 , the second age cohort was followed from age 16 to age 20. Therefore, we were able to examine developmental trajectories from early adolescence until late adolescence.

The attrition was low across the five waves: of the 911 adolescents, $7.8 \%(n=71)$ dropped out at one of the waves. The participants that dropped out did not significantly $(p>0.10)$ differ from participants that continued on gender, age, commitment, balanced relatedness, constructive problem solving, and depression in all five waves. Therefore, we estimated their scores using Full Information Maximum Likelihood within the program Mplus (Muthén and Muthén 2004). Because missing data at each wave were below $8 \%$, we used the same method to impute scores for adolescents having missing data.

Of the present sample, $88.1 \%$ adolescents were of Dutch origin and $11.9 \%$ were ethnic minorities. Forty and a half percent of the adolescents were in high schools preparing for lower level tertiary education or lower level jobs, and $59.5 \%$ were in high schools preparing for college or university. Educational levels of adolescents' fathers and mothers were as follows: $23.1 \%$ and $31.2 \%$ finished only primary or high school, $36.2 \%$ and $39.4 \%$ finished low secondary education, and $40.6 \%$ and $29.4 \%$ finished college or university education, respectively.

\section{Procedure}

Participants came from various high schools in Utrecht and surroundings. Parents and students were invited to participate by providing a letter in which the aims of the study were described and information was given about the option of not participating. Students were required to provide written informed consent. Less than $1 \%$ decided not to participate. Participants completed a series of questionnaires in their classroom after school hours. Research assistants, who attended the administration, gave verbal instructions about filling out the questionnaires and written instructions were also included. Confidentiality of their given answers was guaranteed explicitly. For students who were absent on the day of testing a second assessment time was organized. Students who were absent on both days of testing were not assessed. Each wave, respondents received US\$ 15 after completing the questionnaires.

\section{Measures}

Best Friendships Each individual was asked to nominate a best friend at each wave. Three criteria were used to select adolescents of the total sample $(N=1,331)$. First, 22 individuals that did not nominate a friend on one or more waves were excluded from subsequent analyses. Second, if a friend was mentioned more than once in a particular wave by two or more target adolescents, one target adolescent was randomly selected. Third, of the reciprocated friendships in each wave, one target adolescent was randomly selected to further improve independence of each friendship. This resulted in 911 adolescents whose perceptions were unique in the sense that each friendship was reported on only once. The total group and the selected group of adolescents showed no significant $(p>0.10)$ differences in gender, age, educational level, commitment, balanced relatedness, constructive problem solving, and depression 
in all waves. Both reciprocated $(n=223-254$ across the five waves) and non-reciprocated friends $(n=657-688$ across the five waves) were included in the analyses.

Only a small number of adolescents reported stable friendships $(n=73)$ and even fewer adolescents consistently reported cross-sex friendships $(n=4)$ over all five waves: therefore, friendship stability and gender composition of the friendship dyad were entered as continuous covariates in the analyses. Friendship stability was entered as a continuous variable, ranging from $0=$ stable over 0 waves, $1=$ stable over 2 wave, $2=$ stable over 3 waves, $3=$ stable over 4 waves, to $4=$ stable over five waves. Sixty-three point three percent of the adolescents had same-sex friendships in all waves $(n=577), 18 \%$ had mixed-sex friendships in 1 wave $(n=164), 9.3 \%$ had mixed-sex friendships in 2 waves $(n=85)$, and $9.4 \%$ had mixed-sex friendships in 3 to 5 waves $(n=69)$.

Perceived Commitment Perceived commitment was assessed with the commitment scale of the Investment Model Scale (Rusbult et al. 1998). This scale contains 4 items: "I want the relationship with my best friend to stay good", "I am strongly attached to the relationship with my best friend", "I try my very best to maintain the relationship with my best friend", and "I hope that the relationship with my best friend stays like it is". Participants judged whether the 4 items applied to themselves on a 5-point scale $(1=$ absolutely disagree, 5 = absolutely agree). Cronbach's alphas of commitment ranged between 0.89 and 0.92 from Wave 1 to Wave 5.

Perceived Balanced Relatedness The Balanced Relatedness scale (Shulman et al. 1997b) was used to measure perceived balanced relatedness in best friendships. This questionnaire contained 7 items: "My best friend respects my decisions", "My best friend thinks it is right to sometimes disagree with him/her", "My best friend respects my ideas", "My best friend is not hurt when I have other friends or business", "My best friend allows me to think over my ideas", "My best friend considers my opinion", and "My best friend encourages my suggestions". Participants judged whether the 7 items applied to their best friendship on a 4-point scale $(1=$ absolutely disagree, $4=$ absolutely agree $)$. Cronbach's alphas of balanced relatedness ranged between .88 and .92 from Wave 1 to Wave 5. Correlations between commitment and balanced relatedness range from 0.32 to $0.46(p<0.01)$ within waves, suggesting that commitment and balanced relatedness are distinct but related constructs.

Constructive Problem Solving Perceived constructive problem solving was measured with two subscales of the Conflict Resolution Style Inventory (CRSI) of Kurdek
(1995). The first subscale "Problem Solving" consisted of five items pertaining to constructive problem solvings, such as "Try to find resolutions that are acceptable for the both of us". The second subscale "Engagement" consisted of five items pertaining to non-constructive problem solvings during conflict with best friends, such as "Attacking him/ her personally". These items were recoded so that higher scores reflected more constructive problem solving. Participants were asked to report to what extent these items applied to themselves when having a conflict with their best friend on a 5-point scale ranging from $(1=$ absolutely disagree, $5=$ absolutely agree). An exploratory factor analysis showed that all 10 items loaded higher than 0.40 on one factor at each wave. Furthermore, Confirmatory Factor Analyses (CFA) revealed high factor loadings $(>0.39)$ as well as adequate fit indices $\left(\left(\chi^{2}(16, N=911)>\right.\right.$ $56.19, p<0.01$; CFI's $>0.98$, RMSEA's $<0.04)$ in models in which all items loaded on one single factor. Therefore, for each wave, one score for constructive problem solving was computed. Cronbach's alpha's ranged from 0.82 to 0.85 from Wave 1 to Wave 5 .

Depression Depression was assesed with the revised likertscale version of the Children's Depression Inventory (CDI; Craighead et al. 1998; CDI; Kovacs 1992). This questionnaire contains 27 items. Example items include: "I feel sad all the time", "I often think other people do not like me", and "I hate myself". The items were scored on a 3-point scale, ranging from false, through a bit true, to true. A mean score was computed to create a depression score. The likert-scale version of the CDI is a well-established instrument for measuring depression in non-clinical samples of children and adolescents (Craighead et al. 1998; Kovacs 1992). Cronbach's alphas of this measure ranged from 0.89 to 0.93 from Wave 1 to Wave 5 .

\section{Statistical Analyses}

To answer the first research question, that is, can two different classes be identified based on developmental trajectories of commitment and balanced relatedness across adolescence, we followed the four steps proposed in the step-wise procedure for Growth Mixture Modeling (GMM) (Muthén and Muthén 2000). We first examined univariate longitudinal growth models (LGM) in Mplus (Muthén and Muthén 1998) for commitment and balanced relatedness separately. Different types of latent growth models were estimated to determine which growth model best applied the data, that is, no growth, linear growth, quadratic growth, or freely estimated growth. This was done by comparing RMSEA's, CFI's, and BIC's between each of these models. RMSEA's smaller than 0.05 and CFI's larger than 0.95 
indicate adequate fit of the model, and relatively lower RMSEA's, lower BIC's, and higher CFI's indicate better fit when comparing models (Kline 1998). Intercepts and slopes within the same growth model were correlated because this significantly improved the fit in all models. Gender differences were tested using multiple group models.

An accelerated growth curve design was used to examine commitment and balanced relatedness from age 12 to age 20. This was possible because the younger cohort was followed for five consecutive waves from age 12 until age 16, whereas the older cohort was followed for five consecutive waves from age 16 until age 20. Possible cohort differences in commitment, balanced relatedness, constructive problem solving, and depression were tested by comparing Wave 5 of the younger cohort with Wave 1 of the older cohort (adolescents in both cohorts were then aged 16). A multivariate test $(\mathrm{F}(4,906)=0.94, p>0.05)$ revealed no significant differences in any of the variables between the two cohorts. Therefore, to estimate linear growth across the age cohorts, the loadings on the slope factor for the first age cohort ranged from 0 to 4 for wave 1 to wave 5 , and the same loadings for the second age cohort ranged from 4 to 8 for wave 1 to wave 5 , respectively. Intercepts and slopes were constrained to be equal across age cohorts, resulting in one single developmental pathway for each dimension from age 12 to age 20 .

All models were estimated separately for boys and girls, allowing us to test for different class solutions between boys and girls. Step 2 in this procedure was estimating Latent Class Growth Analysis (LCGA). Several criteria were used to decide on the optimal number of classes (Hill et al. 2000; Muthén and Muthén 2000). First, lower BIC values indicate improvement of that class-solution compared to a class-solution with one fewer class. Second, the usefulness of the classes was determined by examining several indicators: the distinctiveness of the developmental trajectories (based on theoretically expected distinctive trajectories), the predictive value of the class-probabilities (a higher class probability reflecting a better class solution), and the number of adolescents in each class (at least $1 \%$ of all adolescents must be in one class). The third was testing stability of the solutions by repeatedly estimating models with random starting values. Stable models achieve similar solutions despite different starting values. These criteria are also applied in step 3 and step 4 .

In step 3, General Mixture Models (GMM) were estimated that include either the variation of the intercepts or the variation of the slopes within the classes (with the restriction of equal intercept or slope variances across classes). In step 4, General Mixture Models were estimated with both variation in intercepts and slopes. We determined whether unconstraining intercept variances, slope variances, and intercept and slope variances led to a better fit by testing whether the loglikelihood significantly $(p<0.05)$ increased.

To answer research questions 2 and 3, that is, to what extent do adolescents in interdependent and disengaged friendships differ in their development of perceived constructive problem solving and depression, we first examined the univariate longitudinal growth models for constructive problem solving and depression separately to see what growth model fits the data best. Next, General Growth Mixture Modeling was applied (Muthén and Muthén 2000), in which the intercepts and slopes of these two growth models were related to the class-probabilities of friendship types that are based on the development of commitment and balanced relatedness. Thus, the associations between the mean levels and growth over time of perceived constructive problem solving and depression on the one hand and the latent class membership of perceived commitment and balanced relatedness on the other hand were examined. See Fig. 1 for a conceptual model of all three research questions.

\section{Results}

Descriptives of all variables across the five waves can be found in Table 1. We examined the mean levels and changes in commitment and balanced relatedness by testing univariate growth curve models of these two variables (see Table 2). For both dimensions, a linear growth curve (see Figs. 2a and b) fitted the model best, showing lowest $\chi^{2}$ and RMSEA and highest CFI values. Multiple group analyses in which intercept and slope means and variances for boys and girls were unconstrained showed that only the means of the intercepts of both commitment and balanced relatedness differed significantly $(p<0.05)$ with girls scoring significantly higher on perceived commitment and balanced relatedness. Therefore, we estimated the models separately for boys and girls. Whereas the slope of perceived commitment was not significant for boys and girls, the slopes of balanced relatedness was significant and positive for both boys and girls. Note that in all models, variances of intercepts and slopes were significant $(p<$ $0.05)$. This indicates that there are significant individual differences in mean levels and growth of perceived balanced relatedness, commitment, depression, and constructive problem solving.

LCGA and GMM Analyses: Classes in Developmental Trajectories of Commitment and Balanced Relatedness

We first examined step 2, that is, the LCGA-solutions. First, models with four-class solutions showed the lowest BICvalues (BIC's for Class 1 to 4 were 11,041, 10,386, 10,185, 
Fig. 1 Conceptual model of the development of depression and conflict resolution style constructive problem solving associated with perceived friendship class membership Note. $\mathrm{IC}=$ Intercept; $\mathrm{SL}=$ Slope

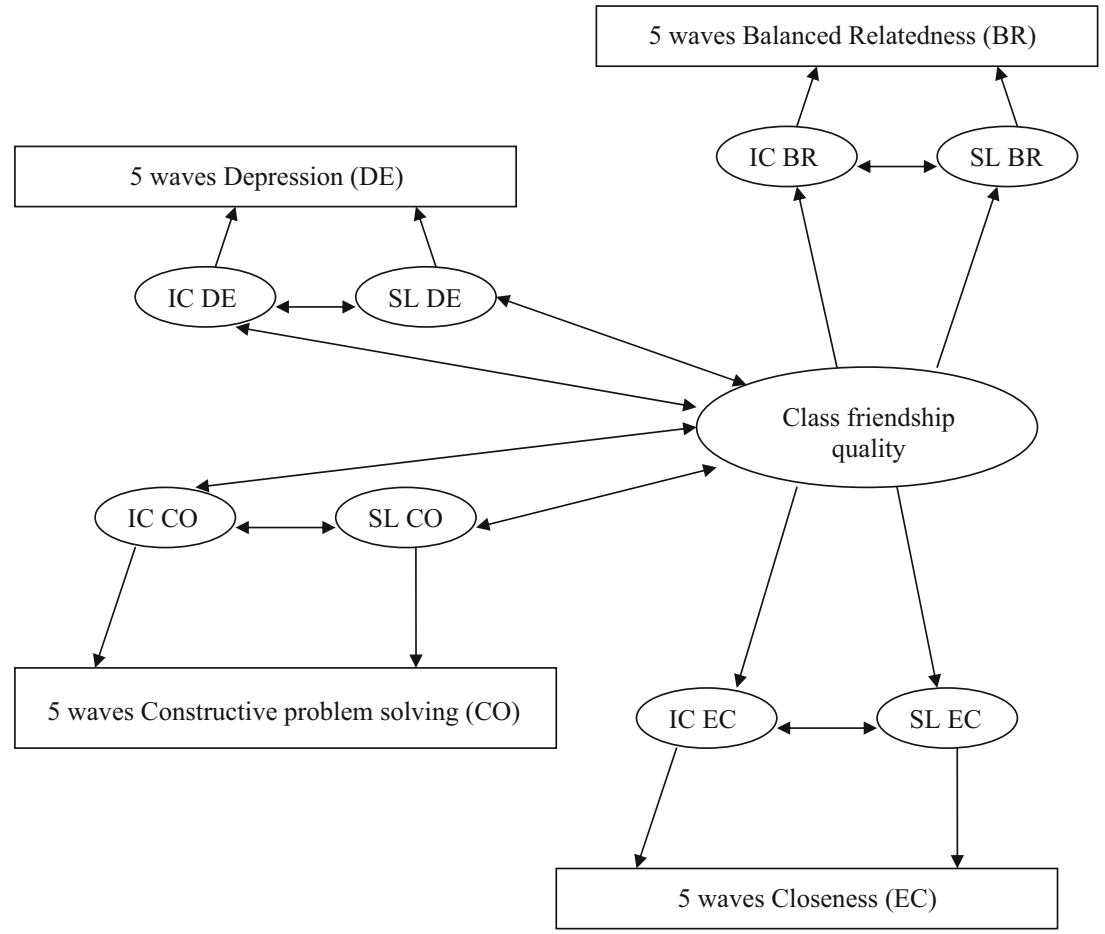

and 10,075 for boys and 10,254, 9,389, 9,296, and 9,221 for girls, respectively). However, this class-solution was not very useful as indicated by a number of issues. The classprobabilities of the fourth class did not differentiate well with the other classes ( $71 \%$ for boys and $73 \%$ for girls) and the number of individuals in this class were few $(n=17$, $3.8 \%$, for boys and $n=21,4.5 \%$, for girls). Furthermore, the growth curve of the fourth class in the four-class solution was very similar in shape to the growth curve of the third class. In addition, increasing the number for starting values resulted in different distributions of adolescents across the classes, indicating that this class solution was not stable. Therefore, we performed step 3 and 4 for the two-class and three-class solution and used loglikelihood to test within each class solution whether the GMM models fitted the data better.

For both the two-class and three-class solution, the model in which variances of intercepts and slopes were unconstrained simultaneously fit the data best. However, although the three-class solution showed a lower BIC value (see above), several other criteria were not met. The classprobabilities of the third class did not differentiate well with the other classes $(70 \%$, for boys and $69 \%$ for girls) and the number of individuals in this class were few $(n=22,4.9 \%$ for boys and $n=15,3.2 \%$ for girls). Furthermore, increasing the number of starting values showed that the three-class solution with free variances of intercepts and slopes was not stable.

The two-class solution with free variances of intercepts and slopes, on the other hand, met all criteria: the number of adolescents in Class 1 ( $n=270,61.1 \%$ for boys; $n=252$, $53.7 \%$ for girls) and Class $2(n=172,38.9 \%$ for boys; $n=$ $217,46.3 \%$ for girls) were large enough to be meaningful, the average class-probabilities were sufficient (Class $1=$ $93 \%, 93 \%$, and Class $2=89 \%, 90 \%$, for boys and girls, respectively), the growth curves were different in mean level and shape and could generally be shaped within the theoretical framework of Shulman and Knafo (to be discussed in more detail in the next section), and different starting values yielded similar distributions of adolescents across the two classes. Finally, loglikelihoods of the models in which variances of intercepts and slopes of commitment and balanced relatedness were unconstrained $(-5,001$ for boys and $-4,455$ for girls, with 24 estimated parameters) were significantly $(p<0.01)$ lower than models in which both variances of intercepts and slopes $(-5,023$ for boys and $-4,582$ for girls, with 20 estimated parameters) were constrained. Therefore, the two-class solution with free variances in intercepts and slopes of commitment and balanced relatedness fitted the data best.

Table 3 shows the means and variances of perceived commitment and balanced relatedness for adolescents in interdependent friendship and adolescents in disengaged friendships by gender. Figure $2 \mathrm{a}$ and Fig. $2 \mathrm{~b}$ depict graphical representations of the corresponding development on these dimensions for the friendship types. We ran repeated measures analyses of variance with commitment and balanced relatedness on all five waves as dependent variables and the four groups (interdependent boys, disengaged boys, interdependent girls, and disengaged 


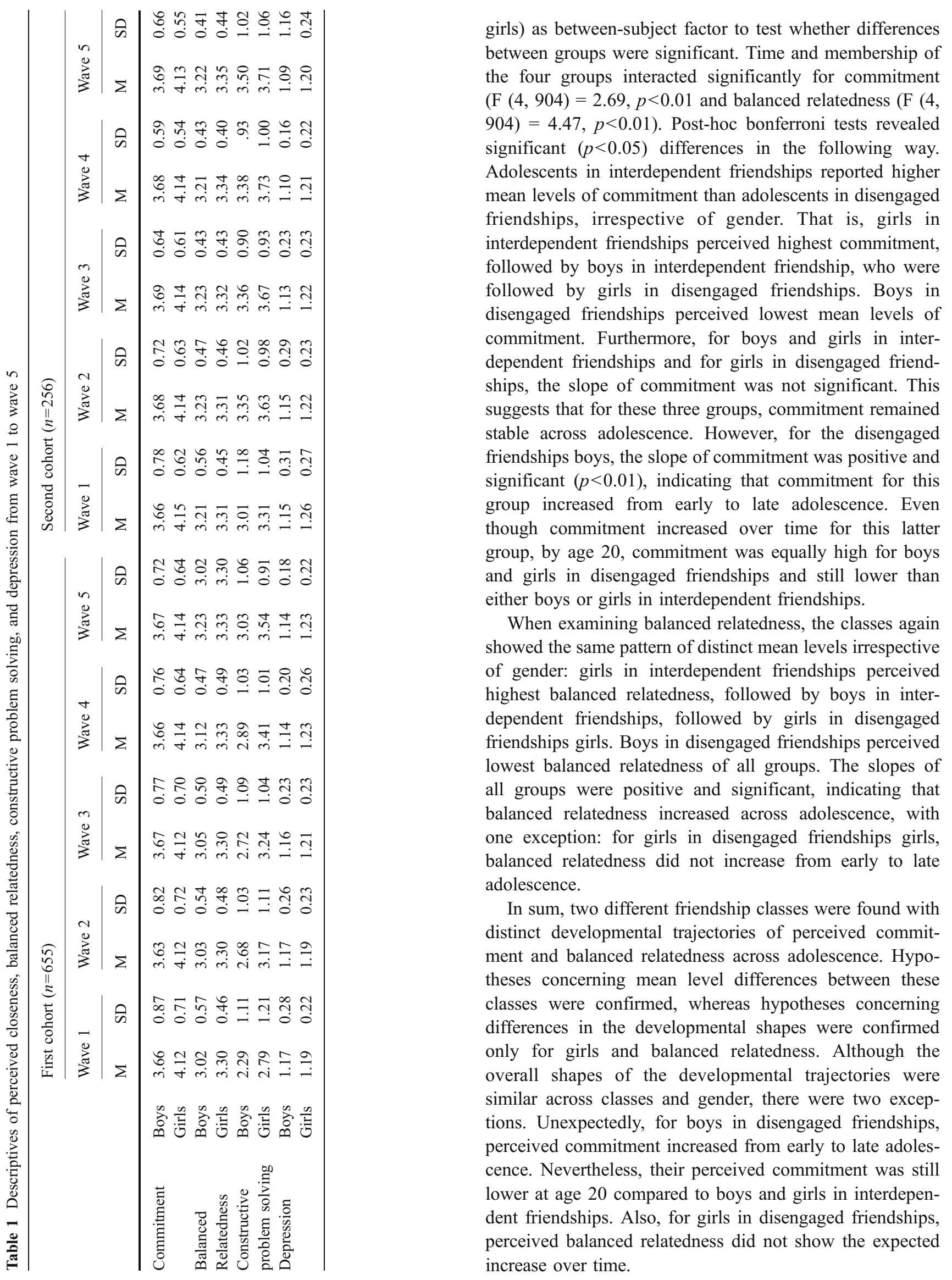


Table 2 Fit indices of final univariate growth curve models

\begin{tabular}{lllll}
\hline Model & $\chi^{2}$ & Df & RMSEA & $C F I$ \\
\hline Males & & & & \\
Commitment & $41.11^{*}$ & 22 & 0.04 & 0.96 \\
Balanced relatedness & $31.26^{*}$ & 22 & 0.03 & 0.96 \\
Depression & $23.93^{*}$ & 22 & 0.02 & 0.99 \\
Constructive problem solving & $42.56^{*}$ & 22 & 0.04 & 0.95 \\
Females & & & & \\
Commitment & $21.76^{*}$ & 22 & 0.00 & 1.00 \\
Balanced Relatedness & $36.05^{*}$ & 22 & 0.04 & 0.97 \\
Depression-Quadratic for Old & $17.30^{*}$ & 15 & 0.02 & 1.00 \\
Constructive problem solving & $47.82^{*}$ & 22 & 0.05 & 0.95 \\
\hline
\end{tabular}

RMSEA $=$ Root mean square error of approximation; CFI = Comparative fit index. All models were estimated with linear growth, unless stated otherwise

$* p<0.05$

In the final distribution of the two-class solution, adolescents were found significantly $\left(\chi^{2}(1, N=911)=17.70\right.$, $p<0.01)$ more often in the disengaged type $(57 \%)$ than in the interdependent type (43\%). Furthermore, gender had a significant effect on class membership $\left(\chi^{2}(1, N=911)=\right.$ $11.15, p<0.01)$, with more adolescents in interdependent friendships among girls (46.3\%) than among boys (38.7\%). The proportion of adolescents in the younger cohort and older cohort did not significantly differ from each other $\left(\chi^{2}(1, N=911)=.04, p>0.10\right)$, indicating that the distribution of adolescents in interdependent and disengaged friendships is equal when comparing younger to older adolescents. Finally, because individuals who kept a reciprocated friendship across all waves $(n=144)$ compared to those who did not $(n=767)$ were equally represented in the two classes $\left(\chi^{2}(1, N=911)=.11, p>0.10\right)$, both reciprocated and unreciprocated friendships were retained in all subsequent analyses.

GMM analyses: Constructive Problem Solving, Depression, and Friendship Type

The univariate growth models of constructive problem solving and depression all showed that linear models fit the data best (see Table 2), with one exception: for depression, quadratic growth fitted the data significantly $(p<0.05)$ better for girls in the older cohort. The slope of constructive problem solving was significant and positive for both boys and girls. The slope of depression was significant and negative for boys, whereas the slope was positive and significant for girls, with the quadratic term being significant and negative for the older cohort of girls only.

Next, GGMM was used to examine the extent to which friendship stability and intercepts and slopes of constructive problem solving and depression are associated with latent class membership. This was done by entering friendship stability and the longitudinal growth models of constructive problem solving and depression in the previous model of friendship type class membership. Table 4 shows the effects of friendship stability and intercepts and slopes of constructive problem solving and depression on latent class membership of friendship type for boys and girls separately. Because gender composition of the friendship dyad was not associated with latent class membership for either boys or girls, loglikelihood and odd ratios for this variable were omitted from this table.

For boys only, a higher stability of friendship was associated with a lower chance of being in the disengaged friendship class. Thus, these results show that boys in disengaged friendships have less stable friendships across adolescence compared to boys in interdependent friendships. For both boys and girls, a higher intercept as well as a higher slope on constructive problem solving were associated with a lower chance of being in the disengaged group. These results indicate that adolescents in disengaged friendships perceive lower mean levels of constructive problem solving, as well as a smaller increase in constructive problem solving across adolescence (see Fig. 2c). Furthermore, for girls, a higher intercept of depression was associated with a higher chance of being in the disengaged group. This suggests that adolescents in disengaged friendships show higher mean levels of depression than adolescents in interdependent friendships across adolescence (see Fig. 2d).

\section{Discussion}

The first aim of the present study was to examine whether interdependent and disengaged friendship types could be found based on the developmental trajectories of perceived commitment and balanced relatedness. This study expands prior knowledge regarding friendship types in adolescence by examining differences in development of perceived commitment and balanced relatedness for these friendship types from age 12 to age 20 using a large sample of adolescents. Results indicate that development of perceived friendship characteristics in adolescence primarily occurs for balanced relatedness and constructive problem solving: for both boys and girls, these characteristics increased across adolescence. Perceived commitment already was relatively high in early adolescence and remained stable across adolescence. Thus, adolescents' cognitive representations concerning friendships seem to become more mature across adolescence with regard to perceived balanced relatedness and constructive problem solving. These findings partly support the theoretical framework proposed by Selman (1990) concerning the development of perceptions of closeness and individuality within friendships: from 
Fig. 2 a Developmental trajectories of commitment for males and females by friendship type. b Developmental trajectories of balanced relatedness for males and females by friendship type. c Developmental trajectories of constructive problem solving for males and females by friendship type. d Developmental trajectories of depression for males and females by friendship type
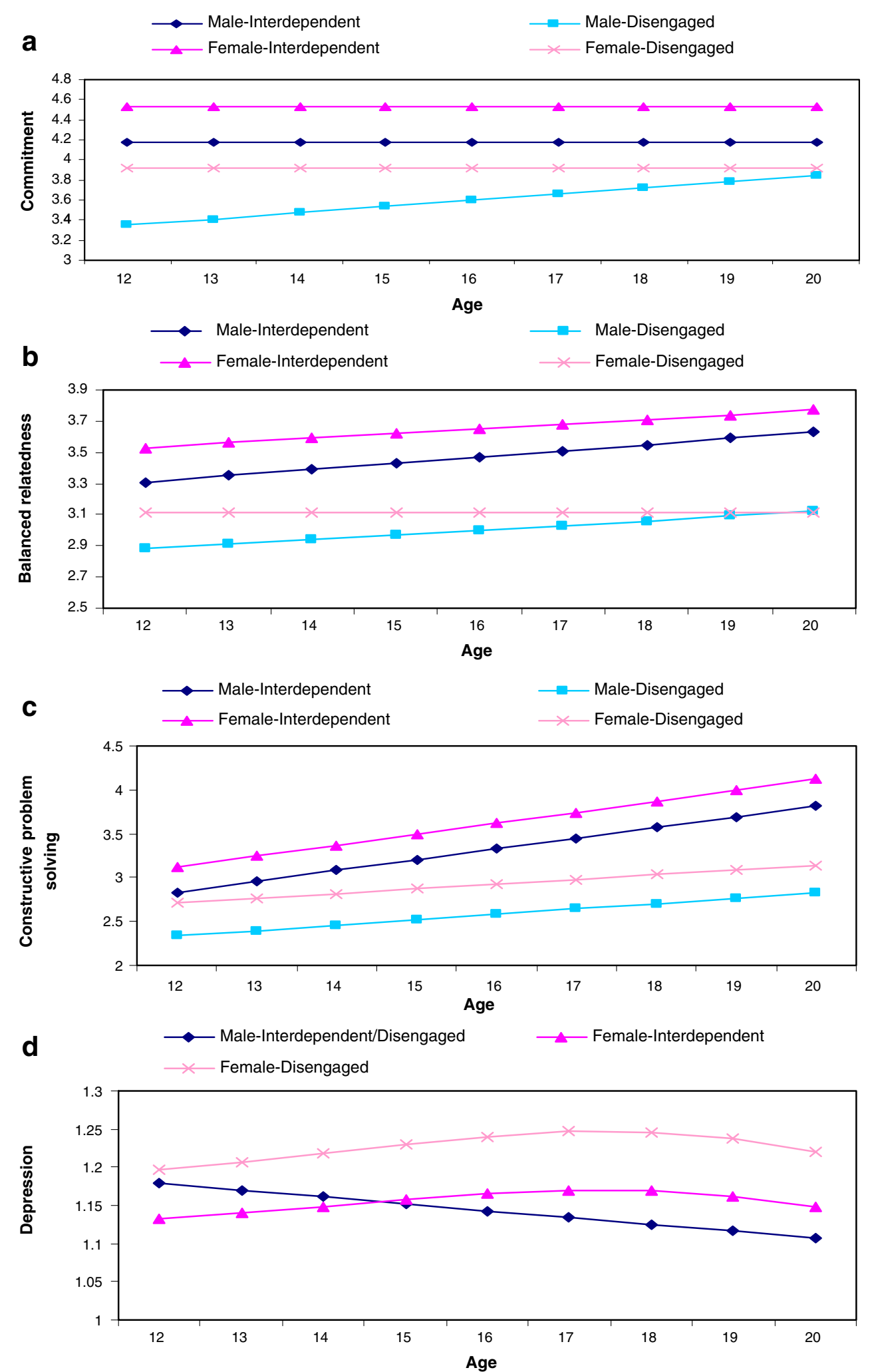

middle adolescence onwards, commitment is expressed by sharing emotions with regard to individuals' own feelings and thoughts as well as friend's feelings and thoughts. Therefore, adolescents perceive that their individuality is relatively more accepted by their friend across adolescence. These results fit within other larger theoretical frameworks dealing with close relationships, such as attachment theory (Bowlby 1973), in which optimal adaptation within close 
Table 3 Means and variances of intercepts and slopes of commitment and balanced relatedness for males and females by friendship type

\begin{tabular}{|c|c|c|c|c|c|c|c|c|}
\hline \multirow[t]{3}{*}{ Dimension } & \multicolumn{4}{|l|}{ Males } & \multicolumn{4}{|l|}{ Females } \\
\hline & \multicolumn{2}{|c|}{ Class 1 (interdependent) } & \multicolumn{2}{|c|}{ Class 2 (disengaged) } & \multicolumn{2}{|c|}{ Class 1 (interdependent) } & \multicolumn{2}{|c|}{ Class 2 (disengaged) } \\
\hline & M & $\Delta$ & M & $\Delta$ & M & $\Delta$ & M & $\Delta$ \\
\hline Intercept commitment & $4.16^{* * *}$ & $0.13 * * *$ & $3.36 * * *$ & $0.13 * * *$ & $4.53 * * *$ & $0.06^{* *}$ & $3.93 * * *$ & $0.06^{* *}$ \\
\hline Slope commitment & 0.00 & $<0.01^{*}$ & $0.05^{* * *}$ & $<0.01 *$ & -0.01 & $<0.01 *$ & $<0.01$ & $<0.01^{*}$ \\
\hline Intercept balanced relatedness & $3.29 * * *$ & $0.04 * *$ & $2.89 * * *$ & $0.04 * *$ & $3.54 * * *$ & $0.03 * *$ & $3.12 * * *$ & $0.03 * *$ \\
\hline Slope balanced relatedness & $0.04 * * *$ & $<0.01 *$ & $0.02 * * *$ & $<0.01 *$ & $0.04 * * *$ & $<0.01 *$ & $<0.01$ & $<0.01^{*}$ \\
\hline
\end{tabular}

${ }^{*} p<0.05,{ }^{* *} p<0.01,{ }^{* * *} p<0.001$

relationships involves use of a partner not only as a source of commitment, but also as a secure base for individual exploration. In addition, these results fit in theoretical frameworks concerning connectedness and individuation (Grotevant and Cooper 1986) that suggest that normative development across adolescence implies maintenance of commitment in the relationship with parents and peers, and is accompanied by increases in individuation. Finally, present findings confirm theories dealing with parenting practices (Baumrind and Black 1967) that suggest that providing an optimal balance between emotional commitment and encouraging individual expression to a certain extent are associated with positive developmental outcomes.

Furthermore, evidence was found for different types of friendships in these developmental trajectories. Replicating and extending previous findings (Shulman 1993, 1995; Shulman and Knafo 1997; Shulman and Laursen 2002), we found two types of friendships with different developmental trajectories of perceived commitment and balanced relatedness from early to late adolescence. Taken together, results largely confirm expectations based on the theoretical models of Shulman and Knafo (1997) concerning adolescents' cognitive representations about friendships. Adolescents in interdependent friendships were characterized by perceiving relatively high, stable commitment in their friendships, while at the same time increasingly perceiving their individuality to be accepted by their best friend. In contrast, adolescents in disengaged friendships were characterized by perceiving stable but relatively low commitment to their best friend and less individuality in their best friendship. The distribution of these two types of friendships was similar to distributions found in prior studies, with around half of all best friendships represented in the interdependent type, and the other half represented in the disengaged type. Unexpectedly, these differences between friendship types emerged from early adolescence onwards instead of starting from middle adolescence onwards. Still, these results are consistent with the notion that individuality becomes an issue in friendships already in early adolescence and continues across adolescence (Laursen 1993).
One unexpected result was that adolescents in interdependent friendships did not show stronger increases in perceived commitment than adolescents in disengaged friendships: in fact, adolescents in interdependent friendships did not increase in perceived commitment across adolescence. Perhaps we did not find further increases in perceived commitment because it was already relatively high for adolescents in interdependent friendships, Similar to this finding, the mean level of perceived social support of best friends has been shown to not change significantly from age 14 to age 16 (Cleveland 2004). Thus, interdependent friends might show high levels of closeness at an earlier age than theoretically expected. Further research is needed to examine whether changes in closeness are found when using different indicators of closeness. For males in disengaged friendships, however, perceived commitment was relatively low, and they increased in perceived commitment and perceived balanced relatedness over time. Nevertheless, these friendship intimacy characteristics were at a mean level comparable to girls in adolescents in disengaged friendships at age 20. In other words, they still

Table 4 General growth mixture model examining friendship stability and intercepts and slopes of constructive problem solving and depression associated with latent class membership of adolescents in disengaged friendships for boys and girls

\begin{tabular}{|c|c|c|c|c|}
\hline & \multicolumn{2}{|l|}{ Boys } & \multicolumn{2}{|l|}{ Girls } \\
\hline & Logit & OR & Logit & OR \\
\hline Stability & $-0.78 * *$ & $0.46^{* *}$ & -0.15 & 0.86 \\
\hline $\begin{array}{l}\text { IC constructive problem } \\
\text { solving }\end{array}$ & $-1.89 * *$ & $0.15^{* *}$ & $-1.86^{* *}$ & $0.16^{* *}$ \\
\hline $\begin{array}{l}\text { SL constructive problem } \\
\text { solving }\end{array}$ & $-1.12 * *$ & $0.33 * *$ & $-1.36^{* *}$ & $0.26^{* *}$ \\
\hline IC depression & 0.62 & 1.86 & $2.24 * * *$ & $9.40 * * *$ \\
\hline SL depression & -0.14 & 0.87 & 1.46 & 4.31 \\
\hline QD depression & -0.92 & 0.40 & -0.32 & 0.73 \\
\hline
\end{tabular}

$\mathrm{IC}=$ Intercept, $\mathrm{SL}=$ Slope, $\mathrm{QD}=$ Quadratic

${ }^{*} p<0.05, * * p<0.01, * * * p<0.001$ 
perceived lower commitment and balanced relatedness compared to adolescents in interdependent friendships. These findings indicate that boys lag in development of commitment compared to girls.

Results furthermore showed that boys were found more often in the disengaged friendship type than girls. This confirms prior results concerning perceptions of friendship quality to typify adolescents in disengaged friendships (Way et al. 2001), and is consistent with findings showing that boys perceive lower friendship quality than girls (e.g., Brendgen et al. 2001). Boys may value multiple relationships in peer groups more than girls (Urberg et al. 1995) and may therefore be found more in adolescents in disengaged friendships than girls. Girls, on the other hand, may invest more time and afford in one particular friendship (Shulman 1993), which explains why they are found more often in adolescents in interdependent friendships than boys.

The second research question concerned differences between adolescents in interdependent and disengaged friendships in the developmental trajectory of constructive problem solving from early to late adolescence. As expected, for all groups, constructive problem solving increased from early to late adolescence (Laursen 1993). Furthermore, interdependent friends showed higher mean levels and greater increases in constructive problem solving compared to disengaged friends. These results are partly consistent with the theoretical model of Selman (Selman 1990), which suggests that from middle adolescence onwards, the increases commitment and balanced individuality results in more constructive conflict solving in which the perspective of both the individual and the friend is taken into account. It might be that because adolescents in disengaged friendships do not integrate each others' opinions when negotiating a conflict as much as adolescents in interdependent friendships, they sustain the feeling that they do not accept each other's individuality while remaining less close to each other. These results furthermore confirm the idea that interdependent and disengaged friendship represent meaningful, distinct categories of friendships in the minds of adolescents, with different ways of experiencing constructive problem solving from early to late adolescence.

The third research question concerned differences between adolescents in interdependent and disengaged friendships in the developmental trajectory of depression. Although depression has been shown to increase from early adolescence until adulthood for girls (e.g., Galambos et al. 2003), in the current study, depression slightly increased from age 12 to around age 17, followed by a decrease from age 17 to 20. This is consistent with findings showing that internalizing problem shows a curvilinear shape across adolescence (Keiley et al. 2003; Lansford et al. 2006). For boys, depression decreased from early to late adolescence, which is consistent with previous findings.

Several prior studies have demonstrated the importance of friendships in the development of adolescent internalizing problems. Specifically, having withdrawn friends (Guroglu et al. 2007), victimization by peers (Hodges et al. 1997), and low friendship quality (Prinstein et al. 2005a) have been associated with higher levels of internalizing problems. The present study confirms that the development of friendship quality and the development of depression are specifically associated for adolescent girls. Although the developmental trajectories of depression were of similar shape for adolescents in interdependent and disengaged friendship types, girls in disengaged friendships showed higher mean levels of depression across adolescence compared to boys and adolescents in interdependent friendships. This difference between friendship types was not found for boys. Girls have been found to exhibit a stronger relational orientation and greater emotional needs in adolescence compared to boys (Rudolph 2002) and it may be that girls are therefore more sensitive to negative exchanges within best friendships (Borelli and Prinstein 2006; Prinstein et al. 2005a). Girls may therefore be more affected by changes in their perceived friendship quality than boys are. In sum, girls in disengaged friendships seem to develop higher levels of depression across adolescence.

Several limitations of the current study should be noted. First, only including the perception of friendship intimacy by the target adolescent, and not the perception of the best friend, limits the findings of the two friendship types only to the perceptions of these adolescents. Future studies should examine the developmental trajectories of commitment and balanced relatedness using perceptions of both friends. Additionally, using observations to examine friendship intimacy might yield different class-solutions, since self-reports and observations of friendship intimacy may expose different characteristics of friendship intimacy. For example, friends typically present a more positive image when using self-reports than is revealed by observations (Poulin et al. 1999). Yet, it may be especially the perception of attitudes or behaviours which are most consequential for adolescent attitudes and behaviours instead of the actual attitudes or behaviours. Third, since the number of stable friendships and mixed-sex friendships was too small to use in the GMM-analyses, future studies should examine to what extent interdependent and disengaged friendships are found in both stable and unstable friendships as well as in same-sex and mixed-sex friendships. Finally, findings are primarily descriptive in the sense that no claims on causality can be made when discussing the differences between interdependent and disengaged types in the developmental trajectories of depression and constructive problem solving. For example, based on this study, we 
cannot tell whether perceptions of higher friendship intimacy lead to a decrease in depression, or whether higher depression leads to a decrease in perceived friendship intimacy.

In sum, the present study is the first to provide evidence for two types of friendships based on developmental trajectories of perceived commitment and balanced relatedness, which represent meaningful, distinct categories in the minds of adolescents. Adolescents in interdependent friendships seem to balance both individuality and commitment across adolescence, and increasingly develop acceptance for each other's individuality. In contrast, adolescents in disengaged friendships seem to maintain a friendship with lower commitment and lower acceptance for each other's opinions, ideas, and needs. Furthermore, adolescents in disengaged friendships seem to lag in their development of constructive problem solving, which might sustain the low perceived commitment and balanced relatedness in this friendship. Finally, girls in disengaged friendships experience highest levels of depression across adolescence, suggesting that girls in these friendships may be at risk for developing depression, while at the same time depressed girls may develop disengaged friendships.

Open Access This article is distributed under the terms of the Creative Commons Attribution Noncommercial License which permits any noncommercial use, distribution, and reproduction in any medium, provided the original author(s) and source are credited.

\section{References}

Baumrind, D., \& Black, A. E. (1967). Socialization practices associated with dimensions of competence in preschool boys and girls. Child Development, 38, 291-327. doi:10.2307/ 1127295 .

Guroglu, B., Lieshout, C. F. M. v., Haselager, G. J. T., \& Scholte, R. H. J. (2007). Similarity and complementarity of behavioral profiles of friendship types and types of friends: friendships and psychosocial adjustment. Journal of Research on Adolescence, 17, 357-386. doi:10.1111/j.1532-7795.2007.00526.x.

Borelli, J. L., \& Prinstein, M. J. (2006). Reciprocal, longitudinal associations among adolescents' negative feedback-seeking, depressive symptoms, and peer relations. Journal of Abnormal Child Psychology, 34, 159-169. doi:10.1007/s10802-005-9010-y.

Bowlby, J. (1973). Attachment and loss: Vol. 2. Seperation. New York: Basic Books.

Brendgen, M., Markiewicz, D., Doyle, A.-B., \& Bukowski, W. M. (2001). The relations between friendship quality, ranked-friendship preference, and adolescents' behavior with their friends. MerrillPalmer Quarterly, 47, 395-415. doi:10.1353/mpq.2001.0013.

Cheng, S., \& Chan, A. C. M. (2004). The multidimensional scale of perceived social support: dimensionality and age and gender differences in adolescents. Personality and Individual Differences, 37, 1359-1369. doi:10.1016/j.paid.2004.01.006.
Cleveland, M. J. (2004). Parent and peer influences reconsidered: the convoy of social support model of adolescent substance use. Dissertation Abstracts International: Section B: The Sciences and Engineering, 65.

Coyne, J. C., \& Downey, G. (1991). Social factors and psychopathology: Stress, social support, and coping processes. In M. R. Rosenzweig, \& L. W. Porter (Eds.), Annual review of psychology. (vol. Vol. 42, (pp. 401-425)). Palo Alto, CA: Annual Reviews.

Craighead, W. E., Smucker, M. R., Craighead, L. W., \& Ilardi, S. S. (1998). Factor analysis of the children's depression inventory in a community sample. Psychological Assessment, 10, 156-165. doi:10.1037/1040-3590.10.2.156.

Engels, R. C. M. E., Deković, M., \& Meeus, W. (2002). Parenting practices, social skills and peer relationships in adolescence. Social Behavior and Personality, 30, 3-18. doi:10.2224/sbp.2002.30.1.3.

Furman, W., \& Buhrmester, D. (1992). Age and sex differences in perceptions of networks of personal relationships. Child Development, 63, 103-115. doi:10.2307/1130905.

Galambos, N. L., Barker, E. T., \& Almeida, D. M. (2003). Parents do matter: Trajectories of change in externalizing and internalizing problems in early adolescence. Child Development, 74, 578-594. doi:10.1111/1467-8624.7402017.

Grotevant, H. D., \& Cooper, C. R. (1986). Individuation in family relationships: a perspective on individual differences in the development of identity and role-taking skill in adolescence. Human Development, 29, 82-100.

Grotevant, H. D., \& Cooper, C. R. (1998). Individuality and connectedness in adolescent development: Review and prospects for research on identity, relationships, and context. In E. E. A. Skoe, \& A. L. Von der Lippe (Eds.), Personality development in adolescence: A cross-national and life span perspective (pp. $3-$ 37). Florence, KY, US: Taylor \& Frances/Routledge.

Hale, W. W. (2001). Behavioral social support between remitted depressed patients with partners and strangers. Journal of Affective Disorders, 64, 285-289. doi:10.1016/S0165-0327(00) 00231-7.

Hill, S. Y., Shen, S., Locke, J., Lowers, L., Steinhauer, S., \& Konicky, C. (2000). Developmental changes in postural sway in children at high and low risk for developing alcohol-related disorders. Biological Psychiatry, 47, 501-511. doi:10.1016/S0006-3223 (99)00175-4.

Hodges, E. V. E., Malone, M., \& Perry, D. G. (1997). Individual risk and social risk as interacting determinants for victimization in the peer group. Developmental Psychology, 33, 1032-1039. doi:10.1037/0012-1649.33.6.1032.

Keiley, M. K., Lofthouse, N., Bates, J. E., Dodge, K. A., \& Pettit, G. S. (2003). Differential risks of covarying and pure components in mother and teacher reports of externalizing and internalizing behavior across ages 5 to 14. Journal of Abnormal Child Psychology, 31, 267-283. doi:10.1023/A:1023277413027.

Kiesner, J., Poulin, F., \& Nicotra, E. (2003). Peer relations across contexts: individual-network homophily and network inclusion in and after school. Child Development, 74, 1328-1343. doi:10.1111/1467-8624.00610.

Kline, R. B. (1998). Principles and practice of structural equation modeling. London: The Guilford.

Kovacs, M. (1992). Children's depression inventory, cdi manual. North Tonawanda (NY): Multi-Health Systems.

Kurdek, L. A. (1995). Predicting change in marital satisfaction from husbands' and wives' conflict resolution styles. Journal of Marriage and the Family, 57, 153-164. doi:10.2307/353824.

Lansford, J. E., Malone, P. S., Stevens, K. I., Dodge, K. A., Bates, J. E., \& Pettit, G. S. (2006). Developmental trajectories of externalizing and internalizing behaviors: Factors underlying resilience in physically abused children. Development and Psychopathology, 18, 35-55. doi:10.1017/S0954579406060032. 
Laursen, B. (1993). The perceived impact of conflict on adolescent relationships. Merrill-Palmer Quarterly, 39, 535-550.

Laursen, B., Finkelstein, B. D., \& Betts, N. T. (2001). A developmental meta-analysis of peer conflict resolution. Developmental Review, 21, 423-449. doi:10.1006/drev.2000.0531.

Muthén, B., \& Muthén, L. K. (1998). Mplus user's guide (4th ed.). Los Angeles, CA: Muthén \& Muthén.

Muthén, B., \& Muthén, L. K. (2000). Integrating person-centered and variable-centered analyses: Growth mixture modeling with latent trajectory classes. Alcoholism, Clinical and Experimental Research, 24, 882-891. doi:10.1111/j.1530-0277.2000. tb02070.x.

Muthén, L. K., \& Muthén, B. O. (2004). Mplus. Statistical analyses with latent variables. User's guide (vol. 3.0). Los Angeles: Author.

Poulin, F., Dishion, T. J., \& Haas, E. (1999). The peer influence paradox: friendship quality and deviancy training within male adolescent friendships. Merrill-Palmer Quarterly, 45, 42-61.

Prinstein, M. J., Borelli, J. L., Cheah, C. S. L., Simon, V. A., \& Aikins, J. W. (2005a). Adolescent girls' interpersonal vulnerability to depressive symptoms: a longitudinal examination of reassuranceseeking and peer relationships. Journal of Abnormal Psychology, 114, 676-688. doi:10.1037/0021-843X.114.4.676.

Prinstein, M. J., Cheah, C. S. L., \& Guyer, A. E. (2005b). Peer victimization, cue interpretation, and internalizing symptoms: preliminary concurrent and longitudinal findings for children and adolescents. Journal of Clinical Child and Adolescent Psychology, 34, 11-24. doi:10.1207/s15374424jccp3401_2.

Reis, H. T., \& Shaver, P. (1988). Intimacy as an interpersonal process. In S. Duck, D. F. Hay, S. E. Hobfoll, W. Ickes, \& B. M. Montgomery (Eds.), Handbook of personal relationships: Theory, research and interventions (pp. 367-389). Oxford, England: Wiley \& Sons.

Rose, A. J., Carlson, W., \& Waller, E. M. (2007). Prospective associations of co-rumination with friendship and emotional adjustment: considering the socioemotional trade-offs of corumination. Developmental Psychology, 43, 1019-1031. doi:10.1037/0012-1649.43.4.1019.

Rudolph, K. D. (2002). Gender differences in emotional responses to interpersonal stress during adolescence. The Journal of Adolescent Health, 30, 3-13. doi:10.1016/S1054-139X(01)00383-4.

Rudolph, K. D., \& Hammen, C. (1999). Age and gender as determinants of stress exposure, generation, and reactions in youngsters: a transactional perspective. Child Development, 70, 660-677. doi:10.1111/1467-8624.00048.

Rudolph, K. D., Hammen, C., Burge, D., Lindberg, N., Herzberg, D., $\&$ Daley, S. E. (2000). Toward an interpersonal life-stress model of depression: the developmental context of stress generation. Development and Psychopathology, 12, 215-234. doi:10.1017/ S0954579400002066.

Rusbult, C. E., Martz, J. M., \& Agnew, C. R. (1998). The investment model scale: measuring commitment level, satisfaction level, quality of alternatives, and investment size. Personal Relationships, 5, 357-391. doi:10.1111/j.1475-6811.1998.tb00177.x.

Selman, R. L. (1990). Fostering intimacy and autonomy. In W. Damon (Ed.), Child development today and tomorrow (pp. 409-435). San Francisco: Jossey-Bass.

Shulman, S. (1993). Close friendships in early and middle adolescence: typology and friendship reasoning. In B. Laursen (Ed.), Close friendships in adolescence (pp. 55-71). San Francisco, CA, US: Jossey-Bass.

Shulman, S. (1995). Typology of close friendships, relationship models, and friendship reasoning in early adolescence. In S. Shulman (Ed.), Close relationships and socioemotional development (pp. 109-127). Westport, CT, US: Ablex.

Shulman, S., \& Knafo, D. (1997). Balancing closeness and individuality in adolescent close relationships. International Journal of Behavioral Development, 21, 687-702. doi:10.1080/ 016502597384622.

Shulman, S., \& Laursen, B. (2002). Adolescent perceptions of conflict in interdependent and disengaged friendships. Journal of Research on Adolescence, 12, 353-372. doi:10.1111/1532-7795.00037.

Shulman, S., Laursen, B., Kalman, Z., \& Karpovsky, S. (1997a). Adolescent intimacy revisited. Journal of Youth and Adolescence, 26, 597-617. doi:10.1023/A:1024586006966.

Shulman, S., Laursen, B., Kalman, Z., \& Karpovsky, S. (1997b). Adolescent intimacy revisited. Journal of Youth and Adolescence, 26, 597-617. doi:10.1023/A:1024586006966.

Stice, E., Ragan, J., \& Randall, P. (2004). Prospective relations between social support and depression: differential direction of effects for parent and peer support? Journal of Abnormal Psychology, 113, 155-159. doi:10.1037/0021-843X.113.1.155.

Sullivan, H. S. (1953). The interpersonal theory of psychiatry. Oxford, England: Norton \& Co.

Urberg, K. A., Degirmencioglu, S. M., Tolson, J. M., \& Hallidayscher, K. (1995). The structure of adolescent peer networks. Developmental Psychology, 31, 540-547. doi:10.1037/0012-1649.31.4.540.

Way, N., Cowal, K., Gingold, R., Pahl, K., \& Bissessar, N. (2001). Friendship patterns among african american, asian american, and latino adolescents from low-income families. Journal of Social and Personal Relationships, 18, 29-53. doi:10.1177/ 0265407501181002. 